\section{$Y$ Chromosomes and} Quinacrine Fluorescence Technique

SIR,-The observation that part of the long arm of the $\mathrm{Y}$ chromosome fluoresces brightly with quinacrine mustard 2 has led Pearson, Bobrow, and Vosa, ${ }^{3}$ using quinacrine hydrochloride, to the discovery that a fluorescent body presumably derived from the $Y$ chromosome is present in the nuclei of epithelial cells in buccal smears, and of cultured lymphocytes, from male subjects. Barlow and Vosa ${ }^{4}$ have observed a similar body in a proportion of spermatozoa and Pearson and Bobrow ${ }^{5}$ using this technique have shown that it is the short arm of the $\mathrm{Y}$ that associates with the $\mathrm{X}$ during first meiotic prophase.

Any test for the presence of $Y$ chromosomes in interphase cells is complementary to the sex chromatin test and has potentialities of similar magnitude. Using $0.5 \%$ quinacrine (mepacrine hydrochloride B.P.) and following the method of Pearson et al. ${ }^{3}$ I have found this to be a relatively simple technique applicable to material prepared in a variety of ways. In the male a "Y chromatin" fluorescent body can be demonstrated in a number of different celltypes. Thus, in small lymphocytes, which of course are unsuitable for the sex chromatin test, the $Y$ chromatin can easily be seen as a peripherally-situated body separated from the rest of the nucleus by a relatively clear area (Fig. 1). On the basis of the presence

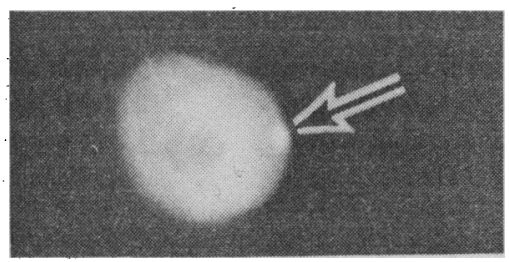

Fig. 1. Small lymphocyte from tonsillar tissue (male, aged 6). $\times 2,660$.

of the body in lymphocytes, fibroblasts, epithelial, or endothelial cells I have been able to "sex" correctly coded smears of blood and sputum as well as smears made from tonsillar tissue removed at operation, squashes of normal and malignant tissue fixed in acetic alcohol, and smears prepared for chromosome studies (by air-drying suspended material on to slides after colchicine and hypotonic pretreatment). Smears fixed by freeze-substitution have also proved suitable, the $Y$ chromatin body often being seen in over $75 \%$ of the cells. Material stored for seven years in a domestic deepfreeze unit has not shown any marked diminution in the brightness of the body. As with the sex chromatin test, buccal smears though convenient to obtain are a little less suitable than other materials, especially if the cells are overlaid by bacteria which are of the same order of size as the chromatin body.

Preliminary results have shown that the $Y$ chromatin body is present in the cells of most tumours of males, a similar body not being seen in those of females. The question of the origin of sex chromatin in testicular teratomas being as yet unresolved, particular interest attaches to the presence or absence of $\mathbf{Y}$ chromosomes in the karyotypes of these tumours. I have found that the epithelial cells in two malignant teratomas of the testis (patients aged 22 and 57) contained both sex chromatin and $Y$ chromatin bodies (Fig. 2). Unfortunately, it

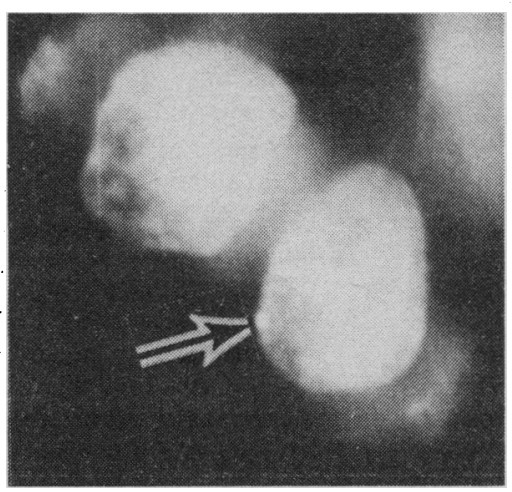

Fig. 2. Group of malignant epithelial cells
testicular teratoma (aged 22 ). $\times 1,660$.

was not possible to stain the cells satisfactorily for sex chromatin after application of the fluorochrome, and it is therefore uncertain whether the same cells contain both bodies. The presence of $\mathbf{Y}$ chromosomes as well as sex-chromatin-forming $\mathbf{X}$ chromosomes in testicular teratomas would clearly have some implications for the genesis of these tumours; thus, their origin from an $\mathrm{X}$-chromosome-containing haploid cell which underwent chromosomal duplication, as proposed by Tavares, $^{6}$ would obviously be ruled out. The presence of a near-triploid modal amount of DNA in the cells of each of the tumours mentioned above is, however, compatible with their origin from a triploid cell with an XXY sex chromosome complement.

A further example of the usefulness of the quinacrine fluorescence technique is provided by a spontaneously-aborted fetus and placenta which, while failing to yield satisfactory chromosome preparations, were found to have a near-triploid DNA content, two sex chromatin bodies in many of the cells, and no $Y$ chromatin body-these together suggesting a $69, \mathrm{XXX}$ karyotype.

This work has been supported by a grant from the Cancer Research Campaign.

-I am, etc.,

N. B. Atrin.

Department of Cancer Research,
Mount Vernon Hospital, Mount Vernon Hospital,
Northwood, Middx.

\section{REFBRENCES}

1 Zech, L., Experimental Cell Research, 1969, 58,

2 George, K. P., Nature, 1970, 226, 80. Nature, i970.' 226,78 ,

Barlow, P., and Vosa, C. G., Nature, 1970, 226, 961.

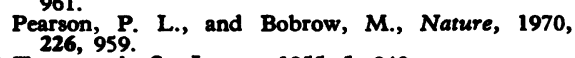
6 Tavares, A. S., Lancet, 1955, 1, 948.

\section{Priapism and Phenothiazines}

SIR,-In an article by Drs. D. Meiraz and J. Fishelovitch ${ }^{1}$ discussing priapism and medication with chlorpromazine the authors state their belief that this medication had not been mentioned in this connexion before. However, I would like to draw readers attention to $\mathrm{my}$ paper $^{2}$ which refers to this topic.

Meiraz and Fishelovitch give details of four cases. One of these patients was diag- nosed as suffering from schizophrenia and one from depression. The other two were described as having been treated with chlorpromazine for a "psychological disorder" and "nervousness." The case I described was diagnosed as suffering from schizophrenia.

In considering these five reported cases it becomes apparent that priapism occurring on chlorpromazine therapy is not confined to schizophrenic patients; it affects both young and old patients; it may follow administration of the chlorpromazine either by injection or via the oral route; withdrawal of chlorpromazine or changing medication to a different phenothiazine seems to result in resolution of the condition; and it is not race-specific.

Meiraz and Fishelovitch suggest that the hypotensive effect of the chlorepromazine may be the basis for development of the priapism. The patient to whom I referred had been intensively investigated and his blood pressure taken many times but, so far as I am aware, at no time was he found to be hypotensive. Also it should be noted that a very large number of patients taking chlorpromazine medication are potentially at risk, yet I know of only five reported cases of the condition. Furthermore, other phenothiazines are known to cause hypotension, yet when I checked with the makers of some of these none knew of a single case of priapism occurring in conjunction with administration of their product.

Nevertheless, as Meiraz and Fishelovitch indicate, priapism has been reported in association with medication by other hypotensive agents. I feel more inclined to support the suggestion of these authors that the explanation lies in an idiosyncratic response of the automatic nervous system rather than central hypotension.

I am grateful to Dr. Rosemary Atherton for drawing my attention to the article by Drs. Meiraz and Fishelovitch, and also to May and Baker, Sandoz, and Smith Kline and French who kindly searched their available literature for me.-I am, etc.,

K. Dawson-Butterworth.

United Sheffield Hospitals,

\section{REFBRENCES}

1 Meiraz, D., and Fishelovitch, J., Israel fournal of Medical Sciences, 1969, 5. 125

2 Dawson-Butterworth, K., British Fournal of Clinical Practice, 1969, 23, 125.

\section{Urinary Tract Infection}

SIR,-Dr. A. J. Wing (26 September, p. 753) highlights some of the problems facing the general practitioner in attempting to assess the significance of symptoms suggesting urinary tract infection encountered on 12 to 20 occasions in every 1,000 consultations ( 17 patients in a personal series of 1,000 consecutive patients last year). There is evidence ${ }^{2}$ that an even higher proportion of patients than the $50 \%$ suggested by Dr. Wing may have no treatable bacteriological cause for symptoms. Such patients cannot on clinical grounds alone be separated from those who need antibacterial treatment. It follows that the general practitioner may feel obliged to take specimens from all patients with symptoms.

The lack of van collecting facilities, communication problems, and insufficiently clear incentives all combine to make this a 